\title{
Adrenal Cortical Response to Immobilization in Conscious and Anesthetized Dogs
}

\author{
Tatuzi Suzuki, Ryozo Higashi, Hiromi Tanigawa, \\ Hiroyuki Ikeda and Kazunori Tamura \\ Department of Physiology (Prof. T. Suzuki), \\ Nagasaki University School of Medicine, Nagasaki
}

\begin{abstract}
Experiments were performed on 9 conscious and 5 anesthetized dogs. The animal was immobilized by strapping supine or prone to the animal table for 60 minutes. The adrenal venous blood was collected before, during and after the period of immobilization and was analyzed for 17-hydroxycorticosteroids (17OHCS). The adrenal 17-OHCS secretion rate in conscious dogs before immobilization was $0.02-0.21 \mu \mathrm{g} / \mathrm{kg} / \mathrm{min}$. In most cases it increased markedly during the immobilization period and reached $0.81-1.80 \mu \mathrm{g} / \mathrm{kg} / \mathrm{min}$. This increase was attributable to elevated 17-OHCS concentration of adrenal venous blood. The 17-OHCS secretion rate in dogs anesthetized with sodium pentobarbital did not show any definite change during and after the immobilization period.
\end{abstract}

Enhanced adrenal cortical activity following immobilization of animals has been assumed from the estimation of the corticosteroid level of peripheral blood. ${ }^{1-3}$ Direct evaluation of adrenal cortical secretion in response to immobilization was made in 2 conscious dogs by Walker and his co-workers. ${ }^{4}$ Their experimental results, however, were not uniform. In one dog, the corticosteroid concentration in adrenal venous blood remained unchanged while the adrenal blood flow increased during immobilization period. In another dog, the adrenal cortical secretion rate was elevated to about twofold as a result of increase in the corticosteroid concentration of adrenal venous blood. Thus, the influence of immobilization upon the adrenal cortical secretion is still obscure.

In the present study, the adrenal 17-hydroxycorticosteroid (17-OHCS) secretion in response to immobilization of animals were directly examined in 9 conscious and 5 anesthetized dogs.

\section{Methods}

Mongrel dogs weighing 11 to $18 \mathrm{~kg}$ were used.

The general procedure for collecting adrenal venous blood from conscious dogs was almost the same as that in our previous paper, ${ }^{5}$ i.e., a modification of the method

Received for publication, October $2,1967$.

This work was supported in part by a grant from the Ministry of Education of Japan. 
of Satake et al. ${ }^{6}$ In order to collect adrenal venous blood from conscious dogs without any evidence of pain, the dorsal spinal roots from the 11th thoracic to the 3rd lumbar spinal cord were sectioned under sodium pentobarbital anesthesia (25 $\mathrm{mg} / \mathrm{kg}$, intravenous). Several weeks after the dorsal spinal root section, the lumboadrenal vein was exposed through the lumbar route, and the side branches of this vein were doubly ligated and cut off between the ligatures. A long silk thread was passed around the lumbo-adrenal vein at the site between the inferior vena cava and the adrenal gland, and a small glass cannula was inserted into the lumbo-adrenal vein lateral to the adrenal gland. A small short rubber tube was connected with a free end of the cannula. The cannula and rubber tube were filled with heparinsaline solution and the rubber tube was clamped. The observation was started usually approximately 18 hours after the adrenal vein cannulation.

In the experiments on anesthetized dogs, the dorsal spinal root sections were omitted. The adrenal vein cannulation was performed under sodium pentobarbital anesthesia (25 mg/kg, intravenous). Approximately 18 hours after the adrenal vein cannulation, the animals were again anesthetized by intravenous injection of sodium pentobarbital in a dose of $25 \mathrm{mg} / \mathrm{kg}$. Anesthesia was maintained by intravenous supplements.

In both series of experiments, the adrenal venous blood was collected by pulling gently the loop of silk thread round the lumbo-adrenal vein and releasing the clamp from the rubber tube. The adrenal venous blood sample collected at each period was $2.5-5.1 \mathrm{ml}$ in volume, and the duration of blood collection was $30-$ 120 seconds. Blood loss was replaced by injection of isotonic saline solution.

Adrenal venous blood samples were taken for the estimation of preimmobilization secretion rate at 30 and 10 minutes before immobilization. The animals were then immobilized by strapping supine or prone to the animal table with a metalic head holder and cotton strings for the limbs. The fastening of animals lasted for 60 minutes. Then the animals were freed from fastening. Adrenal venous blood was collected at 20,40 and 60 minutes after the start of immobilization and at 15 , $30,60,90$ (in 3 cases) and 120 minutes after the end of immobilization period.

The blood samples were centrifuged immediately after collection and the adrenal venous plasma was analyzed for 17-OHCS by the method of Nelson and Samuels. ${ }^{7}$

\section{Results}

The results obtained from the experiments in 9 conscious and $\mathbf{5}$ anesthetized dogs are summarized in Table 1.

\section{Experiments in conscious dogs}

Five dogs (logs 1-5) were fastened supine and the other 4 dogs (dogs 6-9) prone on the animal table. The adrenal 17 -OHCS secretion rates before immobilization were $0.02-0.21 \mu \mathrm{g} / \mathrm{kg} / \mathrm{min}$. 
TABLE 1. The effect of immobilization on adrenal 1\%-hydroxycorticosteroid secretion rate in conscious and anesthetized dogs

\begin{tabular}{|c|c|c|c|c|c|c|c|c|c|c|c|c|c|}
\hline \multirow{3}{*}{$\begin{array}{l}\text { Dog } \\
\text { No. }\end{array}$} & \multirow{3}{*}{$\begin{array}{c}\text { Body } \\
\text { weight } \\
\text { (kg) }\end{array}$} & \multirow{3}{*}{ Sex } & \multirow{3}{*}{ Position } & \multicolumn{10}{|c|}{ Adrenal 17-hydroxycorticosteroid secretion rate $(\mu \mathrm{g} / \mathrm{kg} / \mathrm{min})$} \\
\hline & & & & \multicolumn{2}{|c|}{$\begin{array}{l}\text { Min. before } \\
\text { immobil. }\end{array}$} & \multicolumn{3}{|c|}{$\begin{array}{l}\text { Min. after start } \\
\text { of immobil. }\end{array}$} & \multicolumn{5}{|c|}{$\begin{array}{l}\text { Min. after being freed } \\
\text { from immobil. }\end{array}$} \\
\hline & & & & 30 & 10 & 20 & 40 & 60 & 15 & 30 & 60 & 90 & 120 \\
\hline
\end{tabular}

\begin{tabular}{l|l|l|l|l|l|l|l|l|l|l|l|l|l}
\hline \multicolumn{10}{c}{ Conscious dogs: } \\
1 & 13.4 & $\delta$ & Supine & 0.03 & 0.03 & 0.14 & 0.04 & 0.02 & - & 0.15 & 0.27 & 0.13 & 0.20 \\
2 & 13.4 & $\delta$ & Supine & 0.21 & 0.08 & 1.80 & 1.66 & 1.59 & - & 0.14 & 0.04 & 0.05 & 0.04 \\
3 & 11.5 & $\delta$ & Supine & 0.12 & 0.14 & 1.03 & 1.08 & 0.97 & - & 0.11 & 0.06 & 0.10 & 0.12 \\
4 & 16.0 & $\delta$ & Supine & 0.03 & 0.02 & 1.08 & 1.07 & 1.06 & 0.97 & 0.48 & 0.06 & - & 0.04 \\
5 & 16.7 & $\delta$ & Supine & 0.09 & 0.19 & 0.10 & 0.10 & 1.52 & 0.06 & 0.06 & 0.02 & - & 0.03 \\
6 & 11.4 & $\circ$ & Prone & 0.09 & 0.05 & 0.10 & 0.65 & 0.81 & 0.79 & 0.07 & 0.05 & - & 0.08 \\
7 & 12.5 & $\delta$ & Prone & 0.16 & 0.15 & 0.94 & 1.04 & 1.05 & 0.18 & 0.03 & 0.02 & - & 0.01 \\
8 & 11.4 & $\delta$ & Prone & 0.12 & 0.09 & 1.20 & 1.38 & 1.30 & 0.14 & 0.05 & 0.04 & - & 0.03 \\
9 & 12.3 & $\delta$ & Prone & 0.06 & 0.06 & 1.69 & 1.69 & 1.58 & 0.20 & 0.06 & 0.06 & - & 0.04
\end{tabular}

Dogs anesthetized with sodium pentobarbital:

\begin{tabular}{ll|l|l|l|ll|l|l|l|l|l|l|l} 
& & & & & & \\
10 & 11.4 & $q$ & Supine & 0.15 & 0.09 & 0.05 & 0.12 & 0.09 & 0.09 & 0.06 & 0.04 & - & 0.08 \\
11 & 12.4 & $\&$ & Supine & 0.15 & 0.14 & 0.19 & 0.15 & 0.16 & 0.29 & 0.23 & 0.08 & - & 0.11 \\
12 & 16.1 & $q$ & Supine & 0.08 & 0.04 & 0.03 & 0.05 & 0.03 & 0.12 & 0.14 & 0.03 & - & 0.06 \\
13 & 17.8 & $\delta$ & Prone & 0.04 & 0.02 & 0.04 & 0.08 & 0.07 & 0.11 & 0.06 & 0.05 & - & 0.05 \\
14 & 11.3 & $\delta$ & Prone & 0.14 & 0.05 & 0.07 & 0.10 & 0.06 & 0.10 & 0.07 & 0.10 & - & 0.16
\end{tabular}

Dog 1 was quiet and no obvious excitement was observed during the whole period of immobilization. A decrease in respiration frequency and a slight fall in body temperature were observed. The heart rate, however, increased about 54 beats/min during immobilization. The secretion rates of 17-OHCS during and after immobilization period fluctuated between 0.02 and $0.27 \mu \mathrm{g} / \mathrm{kg} / \mathrm{min}$, i.e., within the range of physiological variation of resting secretion rate.

Dog 5 lay quietly during the first two-thirds of immobilization period. The adrenal 17-OHCS secretion rate was found unchanged at 20 and 40 minutes after the start of immobilization. At 43 minutes, a slight excitement was observed. Then, 2 minutes later the dog began to snarl and struggle violently. These signs of excitement persisted until the end of immobilization period. The respiration became deeper when the animal excited. However, no definite changes in respiration frequency and heart rate were observed. In this case the body temperature showed only a slight rise. A marked increase in adrenal 17-OHCS secretion rate was found at 60 minutes after the start of immobilization. Immediately after being freed from fastening the dog lay down relaxedly and after 15 minutes the secretion rate of $17-\mathrm{OHCS}$ fell down to the previous level.

In $\operatorname{dog} s, 3$ and 4, excitement was observed immediately after fastening. The animals struggled and cried intermittently throughout the period of fastening. The respiration became deeper. Slight increase in the heart rate and respiration frequency was observed. The body temperature rose slightly in dogs 2 and 4 but 
remained unchanged in $\operatorname{dog} 3$. The adrenal 17-OHCS secretion rate increased markedly and its high levels were sustained during the period of fastening. Just after being freed from fastening the animals became quiet. The adrenal 17-OHCS secretion rate returned to the preimmobilization level at 30 minutes after the end of immobilization period in dogs 2 and 3 and at 60 minutes in $\operatorname{dog} 4$.

Dog 6 showed a slight and transitory excitement immediately after the start of immobilization. At 28 minutes the animal began to struggle and cry. Then, excitement occurred from time to time during the period of immobilization. The rate and depth of respiration increased during excitement period. The heart rate and the body temperature remained unchanged in this case. After being freed from immobilization the animal lay quietly on the table. The adrenal 17-OHCS secretion rate at 20 minutes after the start of immobilization did not differ from that before immobilization. At 40 and 60 minutes after the start of fastening, however, the secretion rate rose markedly. An increased secretion rate was also observed at 15 minutes after being freed from fastening. At 30 minutes the secretion rate resumed its resting level.

In dogs 7,8 and 9 , excitement was observed immediately after the start of immobilization. The respiration became deeper. The heart rate, respiration frequency and body temperature rose slightly or remained unchanged. A marked increase in adrenal 17-OHCS secretion rate was observed without exception during the immobilization period. After being freed from fastening the animals became soon quiet, and a rapid decrease in adrenal 17-OHCS secretion rate occurred. At 15 minutes after the end of the immobilization period the secretion rate was found to be within the range of resting secretion rate in all 3 cases.

In the above 8 conscious dogs (dogs 2-9) the adrenal 17-OHCS secretion rate was always increased when the animals were excited by fastening. This acceleration of $17-\mathrm{OHCS}$ secretion rate was attributed without exception mainly to increased 17-0HCS concentration of adrenal venous blood and not to an increase in adrenal blood flow.

\section{Experiments in dogs anesthetized with sodium pentobarbital}

Three dogs (dogs 10-12) were fastened supine and 2 dogs (dogs 13 and 14) prone on the animal table. No symptoms were observed during immobilization period. The heart rate, respiration frequency and body temperature remained unchanged. The adrenal 17 -OHCS secretion rates before, during and after immobilization were $0.02-0.15,0.03-0.19$ and $0.03-0.29 \mu \mathrm{g} / \mathrm{kg} / \mathrm{min}$, respectively. Thus, no definite changes in the adrenal 17-OHCS secretion rate were observed as a result of fastening.

\section{Discussion}

In experiments of Ganong et al., ${ }^{1}$ in which the effect of immobilization on the 17-OHCS level of the plasma was studied, a definite rise in the corticoid level was 
observed whether or not the dogs struggled. In the present study, however, no definite increase in adrenal 17-OCHS secretion was demonstrated in dogs 1,5 and 6 before excitement and struggle. Immobilization did not accelerate the adrenal 17-OHCS secretion rate, unless it caused excitement and struggle of the animal. This was also confirmed by the observation that sodium pentobarbital anesthesia abolished the effect of immobilization on adrenal 17-OHCS secretion.

When marked increase in adrenal 17-OHCS secretion was induced by fastening in the present study, it was mainly due to the elevation of 17-OHCS level in adrenal venous blood. It was not confirmed, however, in any of our experiments that the flucutation of adrenal corticosteroid oatput during immobilization depended mainly on the change in adrenal blood flow as indicated by Walker et al. ${ }^{4}$

\section{References}

1) Ganong, W.F., Gold, N.I. \& Hume, D.M. Effect of hypothalamic lesions on plasma 17 hydroxycorticoid response to immobilization in the dog. Fed. Proc., 1955, 14, 54 .

2) Knigge, K.M. Adrenocortical response to stress in rats with lesions in hippocampus and amygdala. Proc. Soc. exp. Biol. (N.Y.), 1961, 108, 18-21.

3) Betz, D. \& Ganong, W.F. Effect of chlorpromazine on pituitary-adrenal function in the dog. Acta endocr. (Kbh.), 1963, 43, 264-270.

4) Walker, W.F., Zileli, M.S., Reutter, F.W., Shoemaker, W.C. \& Moore, F.D. Factors influencing the 'resting' secretion of the adrenal medulla. Amer. J. Physiol., $1959,197,765-772$.

5) Suzuki, T., Hirai, K., Yoshio, H., Kurouji K-I. \& Yamashita, K. Effect of histamine on adrenal 17-hydroxycorticoid secretion in unanesthetized dogs. Amer. J. Physiol., $1963,204,847-848$.

6) Satake, Y., Sugawara, T. \& Watanabe, M. A method for collecting the blood from the suprarenal gland in the dog, without fastening, narcotizing, laparotomy or provoking any pain. Tohoku J. exp. Med., 1927, 8, 501-534.

7) Nelson, D.H. \& Samuels, L.T. A method for the determination of 17-hydroxycorticosteroids in blood: 17-hydroxycorticosterone in the peripheral circulation. $J$. clin. Endocr., 1952, 12, 519-526. 